\title{
Glycosylation alterations in acute pancreatitis and pancreatic cancer: CA19-9 expression is involved in pathogenesis and maybe targeted by therapy
}

\author{
Ionut Negoi $^{1,2}$, Mircea Beuran ${ }^{1,2}$, Sorin Hostiuc ${ }^{1,3}$, Massimo Sartelli ${ }^{4}$, Alaa El-Hussuna ${ }^{5}$, \\ Enrique de-Madaria ${ }^{6}$
}

${ }^{1}$ Carol Davila University of Medicine and Pharmacy Bucharest, Bucharest, Romania; ${ }^{2}$ Department of General Surgery, Emergency Hospital of Bucharest, Bucharest, Romania; ${ }^{3}$ Department of Legal Medicine and Bioethics, National Institute of Legal Medicine Mina Minovici, Bucharest, Romania; ${ }^{4}$ Department of Surgery, Macerata Hospital, Macerata, Italy; ${ }^{5}$ Department of Surgery, Aalborg University Hospital, Aalborg, Denmark; ${ }^{6}$ Gastroenterology Department, Alicante University General Hospital, ISABIAL, Alicante, Spain

Correspondence to: Ionut Negoi, MD, PhD. Associate Professor of Surgery, Carol Davila University of Medicine and Pharmacy Bucharest, General Surgery Department, Emergency Hospital of Bucharest, No. 8 Floreasca Street, Sector 1, 014461, Bucharest, Romania. Email: negoiionut@gmail.com. Provenance: This is an invited article commissioned by the Section Editor Dr. Le Li (Department of Pancreatic and Biliary Surgery, The First Affiliated Hospital of Harbin Medical University, Harbin Medical University, Harbin, China).

Comment on: Engle DD, Tiriac H, Rivera KD, et al. The glycan CA19-9 promotes pancreatitis and pancreatic cancer in mice. Science 2019;364:1156-62.

Submitted Sep 30, 2019. Accepted for publication Oct 12, 2019.

doi: $10.21037 /$ atm.2019.10.72

View this article at: http://dx.doi.org/10.21037/atm.2019.10.72

\section{Introduction}

Dear editor, we have read with great interest the basic research paper entitled 'The glycan CA19-9 promotes pancreatitis and pancreatic cancer in mice', published recently by Engle et al. in the Science journal (1). This paper underline that CA 19-9 is not only the most used and wellknown tumor biomarker for pancreatic cancer (2), but an active player in the acute pancreatitis and pancreatic cancer pathogenesis, and a potential target for future therapies (1).

For the present editorial we have scrutinized the PubMed/Medline and Web of Science databases, using the search strategy detailed in Table 1. The semantic analysis of the abstracts retrieved in Web of Science may be observed in Figure 1, with inter-relations between 'CA 199 ' 'expression', primary 'tumor', 'borderline' and 'metastatic' tumors.

Pancreatic cancer is associated with an increasing burden between 2007 and 2017, with an increased mortality and disability-adjusted life years (DALYs) during this decade of $24.9 \%$ and $21.6 \%$, respectively (3). Similar patterns were observed for pancreatitis, with $17.6 \%$ increase in mortality and $13.0 \%$ increase in DALYs during the same time interval (3). Acute pancreatitis presented in
2017 an incidence and prevalence of 1300.9 and 118.6 per 1,000 , respectively (4).

The authors investigated the consequences of glycosylation alterations with subsequent CA19-9 elevation in animal models (mouse) and pancreatic organoids. The expression of human FUT3 and 33 GALT5 genes in murine cells generated similar profiles with human CA 19-9 carriers. Induction of CA19-9 expression in mice produced histological signs of acute pancreatitis, such as interstitial edema, lymphocyte infiltrations, and collagen deposition. After 28 days of continuous CA19-9 expression, the disease progressed to chronic pancreatitis, with acinar atrophy, metaplastic ductal cells, and persistent fibro-inflammation (Figure S1) (1). FUT3 expression was associated with removal of the terminal galactose moieties present in rodents but not in humans, and increased levels of Lewis antigens (1).

Glycosylation is a post-translational process, through which carbohydrate molecules or 'glycans' are added to the cell membrane proteins and lipids, with impact in cellular adherence and mobility (5). In patients with pancreatic cancer were observed significant alterations of sialyl Lewis A (CA 19-9), but also of other important glycans, such as sialyl Lewis $\mathrm{X}$ antigen (sLex), truncated O-glycans (Tn 
Table 1 Search strategy used in PubMed/Medline and Web of Science

\begin{tabular}{|c|c|c|}
\hline Search & Query & Items found \\
\hline \#6 & $\begin{array}{l}\text { Search }(((((\text { pancreatic cancer[Title/Abstract]) OR pancreatic ductal adenocarcinoma[Title/Abstract])) OR } \\
\text { pancreatitis[Title/Abstract])) AND ((((Glycosylation alterations[Title/Abstract]) OR CA 19-9[Title/Abstract]) } \\
\text { OR carbohydrate antigen 19-9[Title/Abstract]) OR sialyl-Lewis[Title/Abstract])) AND ((pathogenesis[Title/ } \\
\text { Abstract]) OR targeted therapy[Title/Abstract]) }\end{array}$ & 8 \\
\hline \#4 & $\begin{array}{l}\text { Search (((Glycosylation alterations[Title/Abstract]) OR CA 19-9[Title/Abstract]) OR carbohydrate antigen } \\
\text { 19-9[Title/Abstract]) OR sialyl-Lewis[Title/Abstract] }\end{array}$ & 5,786 \\
\hline \#3 & $\begin{array}{l}\text { Search (((pancreatic cancer[Title/Abstract]) OR pancreatic ductal adenocarcinoma[Title/Abstract])) OR } \\
\text { pancreatitis[Title/Abstract] }\end{array}$ & 92,229 \\
\hline \#2 & Search pancreatitis[Title/Abstract] & 57,123 \\
\hline \#6 & \#5 AND \#4 AND \#3 & 55 \\
\hline \#5 & $\begin{array}{l}\# 2 \text { OR \#1 } \\
\text { Indexes=SCI-EXPANDED, SSCl, A\&HCl, CPCI-S, CPCI-SSH, BKCl-S, BKCl-SSH, ESCI, CCR-EXPANDED, } \\
\text { IC Timespan=All years }\end{array}$ & 130,655 \\
\hline \#4 & $\begin{array}{l}\text { TOPIC: (pathogenesis) OR TOPIC: (targeted therapy) } \\
\text { Indexes=SCI-EXPANDED, SSCI, A\&HCI, CPCI-S, CPCI-SSH, BKCI-S, BKCI-SSH, ESCI, CCR-EXPANDED, } \\
\text { IC Timespan=All years }\end{array}$ & 671,302 \\
\hline \#3 & $\begin{array}{l}\text { TOPIC: (Glycosylation alterations) OR TOPIC: (CA 19-9) OR TOPIC: (carbohydrate antigen 19-9) OR TOPIC: } \\
\text { (sialyl-Lewis) } \\
\text { Indexes=SCI-EXPANDED, SSCI, A\&HCI, CPCI-S, CPCI-SSH, BKCI-S, BKCI-SSH, ESCI, CCR-EXPANDED, } \\
\text { IC Timespan=All years }\end{array}$ & 9,871 \\
\hline
\end{tabular}

and sTn), N-glycans, proteoglycans and galectins, or O-GlcNAcylation (6).

This changing of the tumor cells surface increases cell mobility, with extravasation of the circulating tumor cells into tissues using mechanisms similar with leucocytes populating an inflammatory situs (5). Glycans, such as sialyl Lewis $X$ and sialyl Lewis A, significantly interact with the function of E-selectin ligand, transmembrane adhesion molecules expressed on the surface of vascular endothelial cells (5).

In acute pancreatitis (AP), the genetic background may amplify the deleterious effects of predisposing environmental etiological factors (7). The involved genetic pathways may be or not pancreas specific, and activated in the early or late-phase of the disease. In the early stage of the disease are involved the $P R S S$-cationic trypsinogen, SPINK1 - serine protease inhibitor Kazal type 1, CTRCchymotrypsin $C$ (in premature trypsinogen activation) and interleukin genes, antioxidant enzyme genes, ACE genesangiotensin-converting enzyme, $M I F$-migratory inhibitory factor, $i N O S$-inducible nitric oxide synthase, $C O X-2$ -

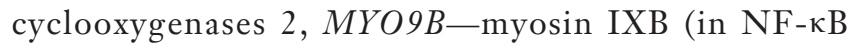
activation). In the late stage of the disease, genetic pathways related to severity are $T L R$ genes-toll-like receptors, 


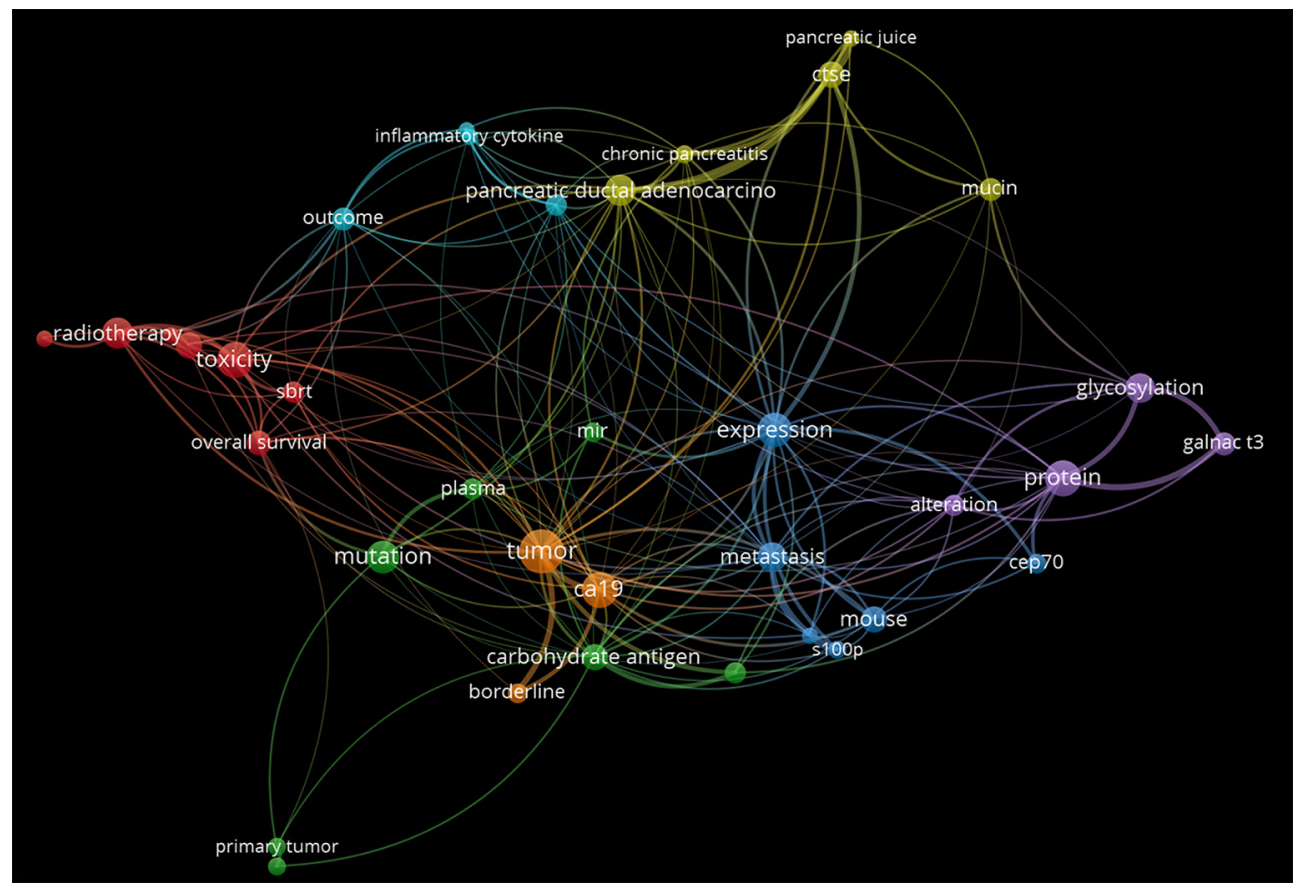

Figure 1 Text analysis of the title and abstracts of papers retrieved in the Web of Science database, using the search strategy from Table 1 , using the VOSviewer software. Maybe observed important inter-relations between 'CA 19-9' 'expression', primary 'tumor', 'borderline' and 'metastatic' tumors as words in the abstracts of the retrieved papers.

CD14, MCP-1 - monocyte chemoattractant protein-1, HBD genes-human $\beta$-defensin 2, and MBL2 - mannose-binding lectin 2. The complications seems to be related to $T N F-\alpha$ genes-tumor necrosis factor- $\alpha, I L-10$-interleukin 10 , and TLR-4-toll-like receptor 4 (7).

On the other hand, the epigenetic regulatory mechanisms play an important role in the control of the inflammatory process in $\mathrm{AP}$, with a rapid and limited spatial increase in the H3K14ac, H3K27ac, H4K5ac and (8).

The micro-RNAs with decreased concentration in AP were miR-92b, miR-10a, and miR-7, while increased concentrations were found for miR-126-5p, miR-148-3p, miR-216a-5p, miR-551b-5p, miR-375 (in patients with severe AP), and miR-216a-5p, miR-551b-5p, and miR-375 in mild AP (9). The miR-21 is an important biomarker for patients with pancreatic cancer (10), but seems to modulate also the inflammatory response during AP, through upregulation of Pias3 and downregulations of Hmgb1 (11).

Engle et al. revealed increased CA19-9 expression in more than $93 \%$ of the resected chronic pancreatitis human specimens, especially at the ductal level and less significant in the acinar compartments (1). Loncle et al. revealed that transition from chronic pancreatitis to pancreatic cancer is promoted by the Il 17, using as a downstream pathway REG33-JAK2-STAT3 inflammatory pathway (12).

CA19-9 expression is associated with hyperactivation of the epidermal growth factor receptor (EGFR) signaling. CA19-9 expression was associated with elevated levels of endogenous fibulin-3 (FBLN3), a glycoprotein with five EGF-like domains, which activated the EGFR pathway (1). Administration of antibodies directed against CA19-9 reduced the inflammatory process of the pancreas, the serum amylase and lipase levels, and decrease the hyperactivation of the EGFR pathway (1). Given the presence of Kras gene mutation in more than $90 \%$ of patients with pancreatic cancer, the role of EGFR, Ras/Raf/MEK/ERK, PI3K/ PTEN/Akt/mTORC1/GSK-3, Janus kinase/Signal Transducer and Activator of Transcription pathways were extensively studied for a better understating of disease pathogenesis (13). The EGFR family of receptors are significantly involved in malignant transformation processes, such as prevention of apoptosis, drug resistance, cancer stem cells and metastasis $(13,14)$. Increasing evidence supports that EGRF signaling is involved the metaplasia process, which converts the acinar cells in progenitor-like ductal cells. This process of acinar to ductal metaplasia may be observed in 
pancreatic cancer initiation in oncogenic Kras and patients with chronic pancreatitis $(15,16)$. EGFR inhibitors, such as gefitinib (Iressa) and erlotinib (Tarceva) block the epithelialto mesenchymal transition, decreasing the metastatic potential of pancreatic cancer cells (14). Some drugs that sensitize the pancreatic cancers cells to EGRF inhibitors were described, such as rhein (17) or alantolactone (18). Inhibition of $\mathrm{PI} 3 \mathrm{~K} \gamma$ significantly reduced acinar cells injury and necrosis in a murine model of AP (19). Karki et al. revealed that Mist1 gene transcription and protein accumulation were significantly reduced during the process of acinar-to-ductal metaplasia found in patients with AP (20). Constitutive mice with expression of Cre-inducible Mist1 transgene (iMIST1) during AP presented a dramatic increase of organ injury secondary to acinar cells death (20).

The CA19-9 expression in mice also harboring Kras oncogene, generates aggressive forms of pancreatic cancer, with anaplastic primary tumors and widespread metastases, associated with decreased median survival (202 versus 460 days) (1). There is a cross talk between Ras and EGFR downstream pathways, which seems to have important role in driving metastasis (13).

The present evidence revealed engineered anti-CA 19-9 antibodies, produced with the aim to specifically target pancreatic tumors and increase the accuracy of imagistic methods (21-23). In a murine study, the authors developed three specifically antibodies $\left({ }^{89} \mathrm{Zr}-{ }^{\text {ss }} \mathrm{DFO}-5 \mathrm{~B} 1\right.$, ${ }^{s s} \mathrm{FL}-5 \mathrm{~B} 1$, and ${ }^{89} \mathrm{Zr}-{ }^{\text {ss }}$ dual-5B1), directed against CA 19-9 for Positron Emission Tomography (PET), near-infrared fluorescent optical imaging and multimodal imaging of pancreatic cancers (24). Currently, a phase 1 study evaluates 89Zr-DFO-HuMab-5B1 (MVT-2163) and HuMab-5B1 (MVT-5873) for tumor imaging using PET scanning in patients with pancreatic cancer and other CA 19-9 positive malignancies (25).

Targeted therapies using $225 \mathrm{Ac}$-labeled tetrazine radioligand and a Trans-cyclooctene-bearing anti CA 19-9 antibodies (5B1) were described for $\alpha$-radioimmunotherapy of pancreatic ductal adenocarcinoma, reducing hematotoxicity while maintaining the therapeutic effects (26).

Human monoclonal antibodies to sialyl-Lewis ${ }^{a}$ were generated and characterized from blood lymphocytes of people immunized with sLe ${ }^{\mathrm{a}}-\mathrm{KLH}$ vaccine (27). The $5 \mathrm{~B} 1$ and 7E3 antibodies increased the median survival of animals engrafted with Colo205 tumor cells. Treatment with 5 B1 antibodies cured $40-60 \%$ of mice, while the mortality was $100 \%$ in untreated mice within 155 days. Both antibodies presented increased activity through a complement-dependent cytotoxicity mechanism; the 5B1 antibody presented also an increased antibody-dependent cytotoxicity (27).

In conclusion, further basic and clinical research is needed in the area of pancreatology, due to the severity of pancreatic diseases and their increased morbidity and mortality. The recent findings revealed the genetic pathways and epigenetic factors involved in pathogenesis of acute pancreatitis, chronic pancreatitis and pancreatic cancer, offering the hope for more precise and effective treatments in patients with pancreatic diseases.

\section{Acknowledgments}

None.

\section{Footnote}

Conflicts of Interest: The authors have no conflicts of interest to declare.

Etbical Statement: The authors are accountable for all aspects of the work in ensuring that questions related to the accuracy or integrity of any part of the work are appropriately investigated and resolved.

\section{References}

1. Engle DD, Tiriac H, Rivera KD, et al. The glycan CA19-9 promotes pancreatitis and pancreatic cancer in mice. Science 2019;364:1156-62.

2. Negoi I, Beuran M, Hostiuc S, et al. Platelet-tolymphocyte ratio and CA19-9 are simple and informative prognostic factors in patients with resected pancreatic cancer. Hepatobiliary Pancreat Dis Int 2019;18:203-5.

3. GBD 2017 Risk Factor Collaborators. Global, regional, and national comparative risk assessment of 84 behavioural, environmental and occupational, and metabolic risks or clusters of risks for 195 countries and territories, 19902017: a systematic analysis for the Global Burden of Disease Study 2017. Lancet 2018;392:1923-94.

4. GBD 2017 Disease and Injury Incidence and Prevalence Collaborators. Global, regional, and national incidence, prevalence, and years lived with disability for 354 diseases and injuries for 195 countries and territories, 1990-2017: a systematic analysis for the Global Burden of Disease Study 2017. Lancet 2018;392:1789-858.

5. Glavey SV, Huynh D, Reagan MR, et al. The cancer 
glycome: Carbohydrates as mediators of metastasis. Blood Rev 2015;29:269-79.

6. Munkley J. The glycosylation landscape of pancreatic cancer. Oncol Lett 2019;17:2569-75.

7. Chen Y, Xie CL, Hu R, et al. Genetic Polymorphisms: A Novel Perspective on Acute Pancreatitis. Gastroenterol Res Pract 2017;2017:5135172.

8. Sandoval J, Pereda J, Pérez S, et al. Epigenetic Regulation of Early- and Late-Response Genes in Acute Pancreatitis. J Immunol 2016;197:4137-50.

9. Xiang H, Tao X, Xia S, et al. Targeting microRNA function in acute pancreatitis. Front Physiol 2017;8:726.

10. Negoi I, Hostiuc S, Sartelli M, et al. MicroRNA-21 as a prognostic biomarker in patients with pancreatic cancer - A systematic review and meta-analysis. Am J Surg 2017;214:515-24.

11. Li X, Lin Z, Wang L, et al. RNA-Seq Analyses of the Role of miR-21 in Acute Pancreatitis. Cell Physiol Biochem 2018;51:2198-211.

12. Loncle C, Bonjoch L, Folch-Puy E, et al. IL17 Functions through the Novel REG3 $\beta$-JAK2-STAT3 Inflammatory Pathway to Promote the Transition from Chronic Pancreatitis to Pancreatic Cancer. Cancer Res 2015;75:4852-62.

13. Fitzgerald TL, Lertpiriyapong K, Cocco L, et al. Roles of EGFR and KRAS and their downstream signaling pathways in pancreatic cancer and pancreatic cancer stem cells. Adv Biol Regul 2015;59:65-81.

14. Beuran M, Negoi I, Paun S, et al. The epithelial to mesenchymal transition in pancreatic cancer: A systematic review. Pancreatology 2015;15:217-25.

15. Ardito CM, Grüner BM, Takeuchi KK, et al. EGF Receptor Is Required for KRAS-Induced Pancreatic Tumorigenesis. Cancer Cell 2012;22:304-17.

16. Navas C, Hernández-Porras I, Schuhmacher AJ, et al. EGF Receptor Signaling Is Essential for K-Ras OncogeneDriven Pancreatic Ductal Adenocarcinoma. Cancer Cell 2012;22:318-30.

17. Yang L, Lin S, Kang Y, et al. Rhein sensitizes human pancreatic cancer cells to EGFR inhibitors by

Cite this article as: Negoi I, Beuran M, Hostiuc S, Sartelli M, El-Hussuna A, de-Madaria E. Glycosylation alterations in acute pancreatitis and pancreatic cancer: CA19-9 expression is involved in pathogenesis and maybe targeted by therapy. Ann Transl Med 2019;7(Suppl 8):S306. doi:10.21037/ atm.2019.10.72 inhibiting STAT3 pathway. J Exp Clin Cancer Res 2019;38:31.

18. Zheng H, Yang L, Kang Y, et al. Alantolactone sensitizes human pancreatic cancer cells to EGFR inhibitors through the inhibition of STAT3 signaling. Mol Carcinog 2019;58:565-76.

19. Lupia E, Goffi A, De Giuli P, et al. Ablation of phosphoinositide 3-kinase- $\gamma$ reduces the severity of acute pancreatitis. Am J Pathol 2004;165:2003-11.

20. Karki A, Humphrey SE, Steele RE, et al. Silencing Mist1 Gene Expression Is Essential for Recovery from Acute Pancreatitis. PLoS One 2015;10:e0145724.

21. Rochefort MM, Girgis MD, Knowles SM, et al. A Mutated Anti-CA19-9 scFv-Fc for Positron Emission Tomography of Human Pancreatic Cancer Xenografts. Mol Imaging Biol 2014;16:721-9.

22. Houghton JL, Zeglis BM, Abdel-Atti D, et al. Pretargeted Immuno-PET of Pancreatic Cancer: Overcoming Circulating Antigen and Internalized Antibody to Reduce Radiation Doses. J Nucl Med 2016;57:453-9.

23. Viola-Villegas NT, Rice SL, Carlin S, et al. Applying PET to broaden the diagnostic utility of the clinically validated CA19.9 serum biomarker for oncology. J Nucl Med 2013;54:1876-82.

24. Houghton JL, Zeglis BM, Abdel-Atti D, et al. Sitespecifically labeled CA19.9-targeted immunoconjugates for the PET, NIRF, and multimodal PET/NIRF imaging of pancreatic cancer. Proc Natl Acad Sci U S A 2015;112:15850-5.

25. Phase 1 Imaging Study of $89 \mathrm{Zr}-\mathrm{DFO}-\mathrm{HuMab}-5 \mathrm{~B} 1$ With HuMab-5B1. Available online: https://clinicaltrials.gov/ ct2/show/study/NCT02687230

26. Poty S, Carter LM, Mandleywala K, et al. Leveraging Bioorthogonal Click Chemistry to Improve 225AcRadioimmunotherapy of Pancreatic Ductal Adenocarcinoma. Clin Cancer Res 2019;25:868-80.

27. Sawada R, Sun SM, Wu X, et al. Human monoclonal antibodies to sialyl-Lewis (CA19.9) with potent CDC, ADCC, and antitumor activity. Clin Cancer Res 2011;17:1024-32. 
A
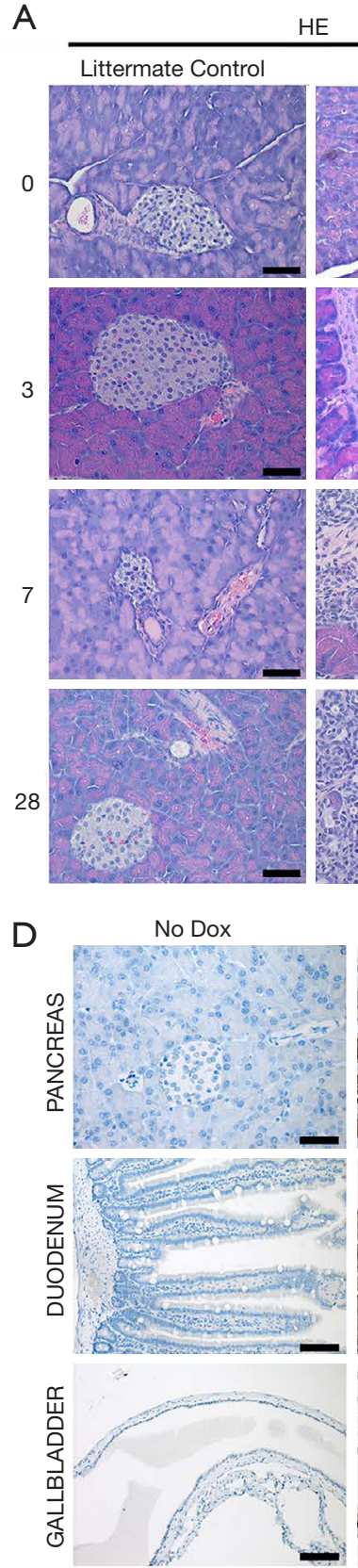

F
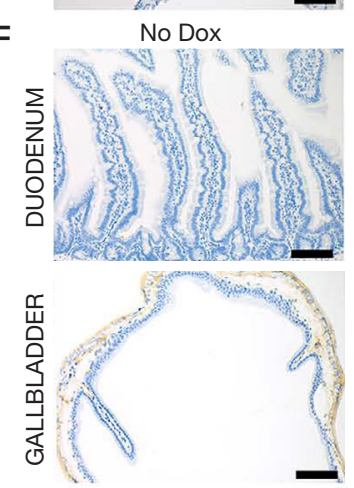

HE
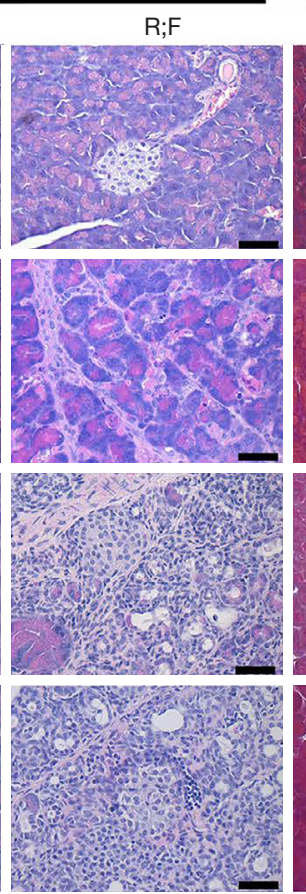

7 Days of Dox
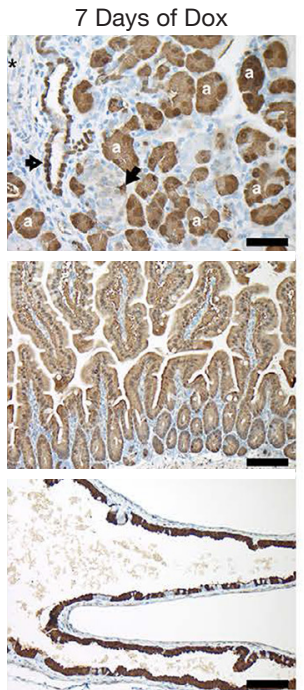

28 Days of Dox
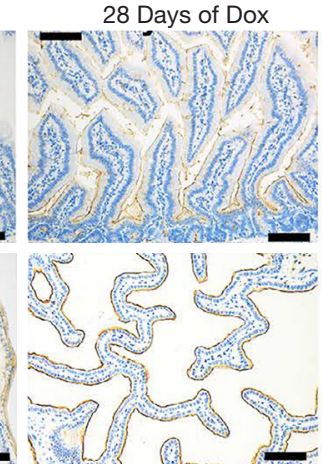

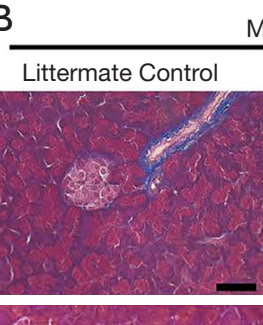

MT

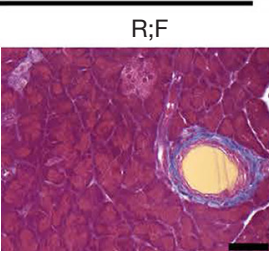

C CA19-9 IHC
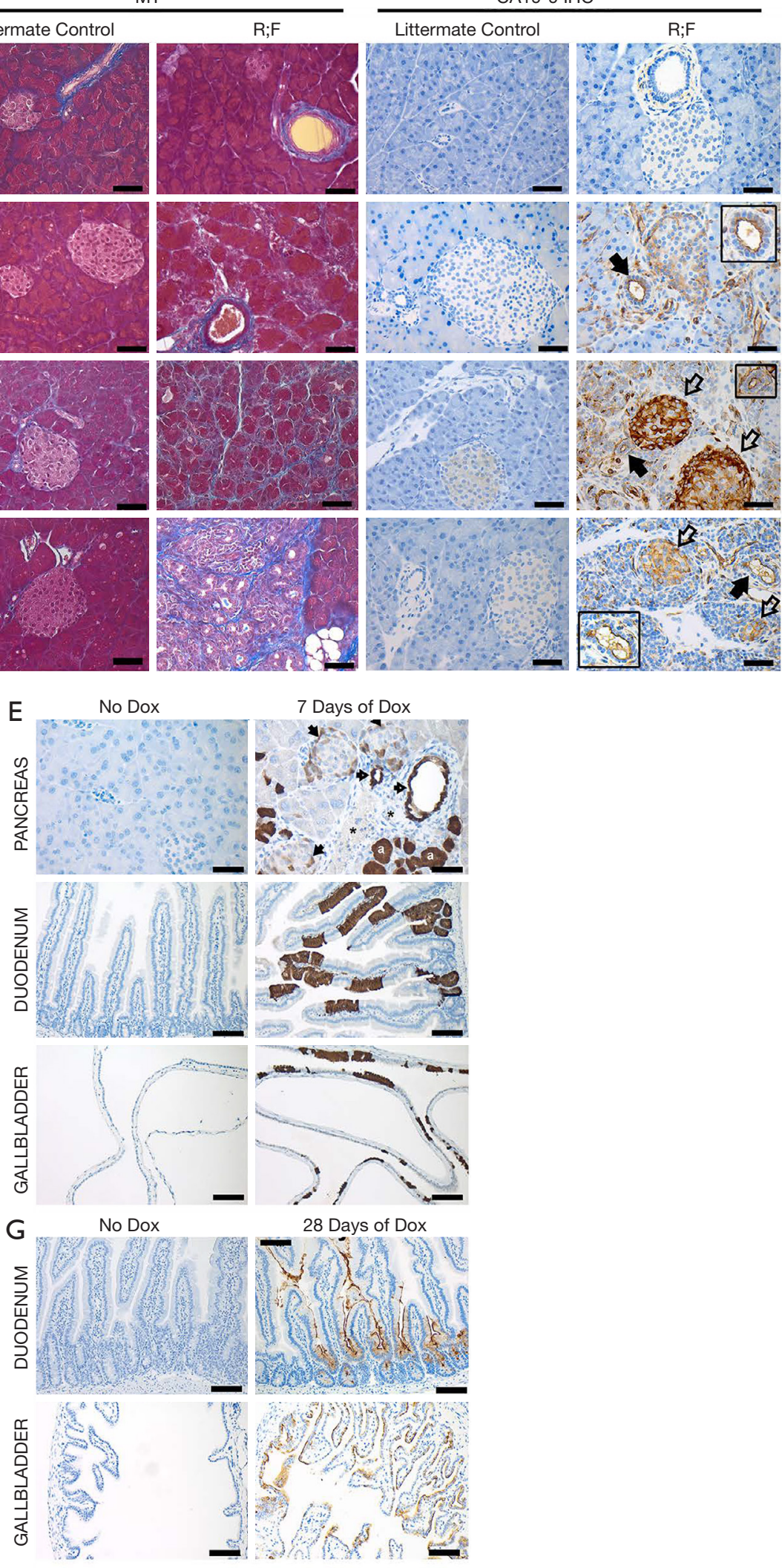

Figure S1 Mouse model comparison for pancreatitis. (A) H\&E, (B) Masson's Trichrome (MT), and (C) CA19-9 IHC of mouse pancreata from genetically negative control littermates and R;F, mice treated with Dox. Closed arrows indicate ducts and open arrows indicate islets. eGFP IHC on untreated and 7-day Dox-treated (D) R;F and (E) C;RLSL;F mouse pancreata, duodenum, and gall bladder. Closed arrows indicate positive islet cells, open arrows indicate positive ducts, * indicates negative vessels and "a" indicates positive acinar cells. CA19-9 IHC on untreated and 28-day Dox treated (F) R;F and (G) C;RLSL;F mouse duodenum and gallbladder. Scale bars =50 $\mu$ m [From ref (1)]. 